http://dx.doi.org/10.22402/j.rdipycs.unam.2.2.2016.68.171-180

\title{
GRADO DE SATISFACCIÓN MARITAL EN RELACIÓN A FACTORES SOCIODEMOGRÁFICOS
}

\author{
Gabriela Taidé Pérez-Solís, María Teresa Dávalos-Romo y Jennifer Rentería-Conseción \\ Universidad Autónoma de Zacatecas Francisco García Salinas \\ México
}

\begin{abstract}
RESUMEN
La insatisfacción marital es un factor que se relaciona con el aumento de divorcios en los últimos años. A nivel nacional, 16 de cada 100 matrimonios terminaron en divorcio. Algunas de las causas que llevan a la insatisfacción marital, la separación de la pareja o al divorcio son la poca demostración de afecto, poca valoración por parte de la pareja, infidelidades, secretismos y manejo de la economía, entre otras. Esta investigación tuvo como objetivo describir los factores sociodemográficos (género, número de hijos, edad, escolaridad y años de casados o de convivencia) asociados con la satisfacción. Para esto, se tuvo la participación de 42 hombres y 58 mujeres, en una relación marital, de concubinato o unión libre, de un contexto semiurbano, como el municipio de Jalpa, Zacatecas. Se utilizó la escala de satisfacción marital. Los resultados sugieren que existen diferencias significativas entre el factor de escolaridad y la satisfacción marital; no se encontraron diferencias estadísticamente significativas al analizar otros factores; los datos obtenidos en este trabajo no apoyan investigaciones previas realizadas en contextos urbanos. Con lo anterior se concluye que la satisfacción marital es multicausal y está asociada al nivel educativo de un individuo.
\end{abstract}

Palabras Clave:

Relaciones de pareja, escolaridad, convivencia, hijos, género.

\section{DEGREE OF MARITAL SATISFACTION IN RELATION TO SOCIODEMOGRAPHIC FACTORS}

\begin{abstract}
The marital dissatisfaction is a factor that is related to the divorces increase in the last years. At national level 16 of every 100 marriages ended in divorce. Some of the causes that lead to the marital dissatisfaction, the separation of the couple or to the divorce infidelities are few demonstration of affection, small evaluation on the part of the couple, secrecy, handling of the economy, between others. This investigation took as a target to describe the factors sociodemographic (genre, number of children, age, schooling and years of married or of coexistence) associated with the satisfaction. For this, there was had the participation of 42 men and 58 women, in a marital relation, of concubinage or free union, of a semiurban context as it is the Municipality of Jalpa, Zacatecas. There was used the Scale of Marital Satisfaction. The results suggest that there exist significant differences between the factor of schooling and the marital satisfaction; differences were not as per statistics significant on having analyzed other factors; the information obtained in this work does not support previous investigations realized in urban contexts. With the previous thing one concludes that the marital satisfaction is for various reasons and is associated at the educational level of an individual.
\end{abstract}

\section{Keywords:}

Relationships, education, cohabitation, children, gender.

Bitácora del Artículo:

| Recibido: 1 de Febrero de 2016 | Aceptado: 1 Marzo de 2016 | Publicado en línea: Julio-Diciembre de 2016 | 


\title{
GRADO DE SATISFACCIÓN MARITAL EN RELACIÓN A FACTORES SOCIODEMOGRÁFICOS
}

\author{
Gabriela Taidé Pérez-Solís, María Teresa Dávalos-Romo y Jennifer Rentería-Conseción
}

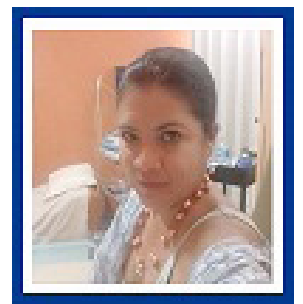

Gabriela Taidé Pérez-Solís

Universidad Autónoma de Zacatecas

Francisco García Salinas

Correo: taide0906@hotmail.com

Licenciada en Psicología Clínica por la Universidad Autónoma de Zacatecas, Campus Jalpa. Actualmente labora en Unidad de Servicios de Apoyo a la Educación Regular "Justo Sierra Méndez" Jalpa, Zacatecas. Educación Especial, Secretaría de Educación Zacatecas.

Ver más...

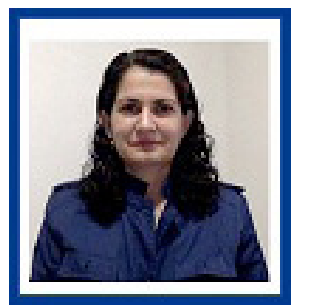

María Teresa Dávalos-Romo Universidad Autónoma de Zacatecas Francisco García Salinas Correo: tt-kiki@hotmail.com

Doctorado y maestría en Psicología, Universidad Autónoma de Madrid. Profesora-investigadora de tiempo completo de la Unidad Académica de Psicología de la Universidad Autónoma de Zacatecas "Francisco García Salinas". Miembro del Sistema Nacional de Investigadores, nivel Candidato.

Ver más...

\section{CONTRIBUCIÓN DE LAS AUTORAS}

Lic. Gabriela Taidé Pérez Solís. Elaboración de manuscrito. | Dra. María Teresa Dávalos Romo. Elaboración y revisión de documento.

\section{AgRADECIMIENTOS}

Las autoras agradecen a la Universidad Autónoma de Zacatecas, Extensión, Jalpa.

\section{Datos de Filiación de las Autoras}

Universidad Autónoma de Zacatecas “Francisco García Salinas”, Campus Jalpa, México.

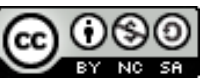

Copyright: (c) 2016 Pérez, G., Dávalos, M. \& Rentería, J.

Este es un artículo de acceso abierto distribuido bajo los términos de la licencia Creative Commons Reconocimiento-NoComercial -Compartirlgual 4.0 Internacional, por lo que su contenido gráfico y escrito se puede compartir, copiar y redistribuir total o parcialmente sin necesidad de permiso expreso de sus autoras con la única condición de que no se puede usar con fines directamente comerciales y los términos legales de cualquier trabajo derivado deben ser los mismos que se expresan en la presente declaración. La única condición es que se cite la fuente con referencia a la Revista Digital Internacional de Psicología y Ciencia Social y a sus autoras. 


\section{TABLA DE CONTENIDO}

INTRODUCCIÓN

MATERIALES Y MÉTODOS

Participantes, 6

Material, 6

Procedimiento, 6

Análisis de datos, 6

Resultados

Discusión

ConcLusiones 


\section{INTRODUCCIÓN}

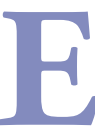
I ser humano es por naturaleza esencialmente social. Aristóteles señala en el año 303 a.C. que la interacción del individuo con los demás se establece como una parte intrínseca de éste, dando cabida a una de las primeras asociaciones de la especie humana -la de marido y mujer-, constituyéndose como la base de la familia (López y García, 2005). En la actualidad, a pesar de los cambios que ha tenido la pareja, y por consiguiente la familia, dichas asociaciones continúan dentro de lo establecido como norma, ya que en casi todas las sociedades se le ha protegido como una forma de asegurar la continuidad de la especie y el progreso de la civilización (Covarrubias y Gómez, 2012). Sin embargo, al transcurrir los años algunas relaciones de pareja poco a poco se van tornando difíciles al atravesar ciertas crisis (Campuzano, 2002). Al constituirse una relación de convivencia en pareja, ya sea como matrimonio, concubinato o unión libre, se adquiere un compromiso de gran magnitud que no se conserva como un estado inalterable, sino que va cambiando con el tiempo y las circunstancias (Becerra, Roldán y Flores, 2012), y no siempre tienen un final de cuento - "y vivieron felices para siempre..."-

Estos cambios pueden impactar en la satisfacción marital, perturbando así el bienestar y estabilidad de la relación de pareja (Becerra et al., 2012); por tanto, la satisfacción marital es considerada como la actitud hacia la pareja normalmente percibida en términos de calidad de la relación (Dainton, Stafford y Canary, 1994), la actitud de mayor o menor favorabilidad hacia la propia relación marital (Roach, Frazier y Bowden, 1981), o la evaluación de aspectos específicos de la vida matrimonial (Stewart, 2012). En este sentido, no es difícil suponer que toda relación de pareja es una dinámica relacional humana, en donde interactúan varios factores que afectan la calidad de vida en pareja, tales como la convivencia entre la díada, la evaluación que hace el individuo de su relación, ingresos y gastos económicos, y las conductas de afecto (Armenta, Sánchez y Díaz, 2012).

Algunos estudios sugieren que el porcentaje de divorcios es alarmante (Castro, 2014); según el INEGI (2014, 2015), señala que del año 2009 al 2014, 16 de cada 100 matrimonios terminó en divorcio, y que a lo largo del primer trimestre del 2015 se han divorciado 609 parejas zacatecanas; sin embargo, el INEGI no documenta la predilección que las parejas manifiestan por las separaciones de hecho que se dan en México (Ojeda y González, 2008), es decir, las parejas se separan, pero no disuelven el vínculo matrimonial de manera legal, lo que implica considerar que las estadísticas de divorcio pueden estar nubladas y ser mayores. Aun así, las estadísticas permiten conocer el fenómeno del divorcio; la destrucción del núcleo familiar puede generar graves repercusiones, como la deserción escolar, la vagancia, la baja autoestima en los niños, la salud e incluso la vida laboral (Arias, 1989).

Ante esto, se han realizado estudios que abordan la relación de satisfacción e insatisfacción marital con factores como el género. Al respecto, Bounchard (2013) sugiere que tanto hombres como mujeres se ven influidos de manera similar en su satisfacción marital por eventos que ocurren durante su vida, como el nacimiento de los hijos. Estos datos coinciden con los aportados por Ottu y Akpan (2011), los cuales encontraron que aun cuando las diferencias de género contribuyen a los problemas maritales, en la muestra estudiada no hay diferencias significativas al correlacionar el género y la satisfacción marital, mostrando que el estar o no maritalmente satisfecho no parece depender del sexo de una persona. Por otra parte, y opuesto a los resultados expuestos, Pick y Andrade (1988) —en un estudio realizado en México- encontraron que existen diferencias entre hombres y mujeres, en el sentido de que la mujer se ve más afectada que el hombre respecto a su propia percepción acerca de los cambios físicos en su cuerpo y a una mayor dedicación al cuidado de los hijos. En este mismo sentido, Lavner y Bradbury (2010) corroboran los hallazgos anteriores, puesto que el tener un matrimonio poco satisfactorio puede ser más preocupante para las mujeres que para los hombres; esto se debe a que la carga de obligaciones no es equitativa. El hombre suele beneficiarse con el matrimonio salvándole del trabajo doméstico o del cuidado de los hijos. Por su parte, las mujeres no reciben estos beneficios en la relación, por lo que se considera que su insatisfacción marital rebasa en mucho a la de los hombres, y en consecuencia puede presentar menos razones para continuar con o permanecer en dicha relación. La cuestión del género no deja del todo claro si existen diferencias significativas entre hombres y mujeres, puesto que no son concluyentes, y ello hace necesario seguir la línea de relación entre estas variables.

Otro factor que se ha documentado es la crianza y el cuidado de los hijos, donde el número de éstos puede afectar la satisfacción marital, sobre todo cuando los hijos requieren mayor atención de los padres y se descuidan los aspectos que fortalecen la vida marital, causando malestar en uno o en ambos miembros de la pareja (Shapiro, Gottman y Carrere, 2000). Algunas investigaciones sugieren que la satisfacción marital varía si la pareja tiene 
o no descendencia, incluso influyendo la cantidad que han decido procrear, pues según sea la cantidad la pareja invierte más en la organización, dedicación y tiempo. Ante esto, no es difícil suponer que la satisfacción marital tienda a disminuir, mostrando mayor insatisfacción (Pick y Andrade, 1988; Mora, Gómez y Rivera, 2013). Cabe destacar que actualmente son varias las parejas que han decidido no tener hijos; Sandler (2013) menciona que las principales razones son la económica, el esfuerzo y la dedicación que requiere el criar y educar a un niño; en Estados Unidos las mujeres exitosas pierden salario, promociones y beneficios si deciden tener hijos, por lo cual - de forma personal o en pareja- posponen o suprimen la maternidad-paternidad en favor del trabajo o la educación, generando ambos un estilo de vida satisfactorio. Este mismo autor encuentra que esta tendencia se está extendiendo poco a poco a otros grupos demográficos, como el de los latinos y los europeos.

La edad es otro factor relacionado con la satisfacción marital; esto se muestra en estudios realizados por Vera, Félix-Castro y Rodríguez-Barrera (2001) y Martínez (2004), donde se encontró que los efectos de la edad en la satisfacción marital tiene una relación positiva, es decir, a mayor edad de la pareja, ésta se sentirá más satisfecha, adaptándose poco a poco a su nuevo rol, las expectativas matrimoniales sufren cambios favorables respecto a las que se tenían al inicio de la relación, existe más conciencia de los problemas, y por tanto la pareja suele preocuparse y ocuparse más por los aspectos tanto maritales como familiares, aun cuando ambas partes asuman una mayor responsabilidad en la relación.

Por otro lado, en el discurrir de la vida marital, conforme se acumulan los años de casados, se ha considerado que las parejas pasan por ciertas etapas. Campuzano (2002) y Martínez (2004) coinciden en señalar que las parejas jóvenes, en sus primeros años de matrimonio, atraviesan una etapa donde la insatisfacción marital es consecuencia de conflictos inducidos por la inmadurez de los cónyuges y las expectativas poco realistas que tienen uno del otro, lo que les impide salir de la relación o trascenderla progresivamente; para las parejas situadas en la etapa media, los conflictos maritales se dan por las características del crecimiento individual, que requieren una revisión de los logros y de posibles cambios futuros; en la etapa en que se ubican las parejas maduras, hay una aceptación y compromiso para con el otro, pero hay también una aceptación de que hay anhelos irrealizables y se conforman con lo que obtienen de la relación; en este caso los conflictos generadores de la insatisfacción marital suelen ser pocos o nulos. Según Kimble (2002), a través de los años las etapas de una relación marital adquie- ren un patrón en forma de " $U$ ", donde el nivel máximo de satisfacción marital se presenta en los primeros años, para decaer en los años intermedios y volver a elevarse en el periodo final de la pareja. En este mismo sentido, Rivera y Heresi (2011) señalan que conforme aumentan los años de matrimonio, aumenta la satisfacción marital. Por el contrario, Pick y Andrade (1988) consideran que este patrón es progresivo lineal en declive.

En cuanto al factor de escolaridad, se ha encontrado que las personas con escolaridad baja y media superior muestran una mayor insatisfacción en su matrimonio; las personas que tienen un bajo nivel escolar suelen desempeñar trabajos de poco prestigio que les reditúan ingresos insuficientes para solventar las necesidades básicas de la familia, mientras que los de nivel medio realizan comparaciones con los sujetos con mayor preparación profesional, generando una insatisfacción marital como reflejo de una insatisfacción general (Arias, 1989; Vera, 2011). En cuanto a los individuos con un alto nivel de escolaridad, Campbell, Converse y Rodgers (1976) encontraron que las personas que perciben un ingreso alto regularmente son quienes tienen una educación profesional, lo cual se refleja en una satisfacción marital alta.

Por lo anterior, se sugiere que son múltiples y variados los factores involucrados en la satisfacción marital; además, se pueden incluir la intimidad, el compromiso, la comunicación, la congruencia, la orientación religiosa, la reciprocidad y el amor, así como el entendimiento, la paciencia y la responsabilidad (Armenta y Díaz-Loving, 2008), la comunicación, la insatisfacción sexual, la ansiedad global, el compartir tiempo juntos y los convencionalismos sociales (Snyder, 2008). Todos y cada uno de ellos fueron estudiados en distintos contextos urbanos, pero no semiurbanos o rurales. Por ello, se considera relevante el estudio en las relaciones de pareja, ya que a pesar de su diversidad y complejidad, así como del paso del tiempo y los cambios de paradigma que sufre la familia como institución, el matrimonio sigue siendo el elemento principal de toda sociedad, y en consecuencia es de gran importancia destacar que cuando una pareja se encuentra en armonía influye de manera positiva en la salud física y psicológica de todos los miembros de la familia, además de que si existe una alta probabilidad de que al identificar los factores de la interacción matrimonial que originan una mayor insatisfacción marital podrían atajarse sus efectos (Wilson, 1998).

El objetivo de este trabajo fue determinar si hay diferencias significativas en factores sociodemográficos - como el género, número de hijos, edad, escolaridad y número de años de casados- que se asocian con la satisfacción marital de parejas casadas, en concubinato 
o unión libre, en un contexto semiurbano como el municipio de Jalpa, Zacatecas, México.

\section{Materiales y métodos}

\section{Participantes}

El tipo de muestreo utilizado fue no probabilístico por conveniencia (Bradley, 1999), captado en un momento previo a una serie de pláticas para parejas en cinco escuelas primarias del municipio de Jalpa, Zacatecas; la muestra se conformó de 100 personas: 58 mujeres (58\%) y 42 hombres (42\%), con edades de entre los 17 y los 59 años. La participación estuvo sujeta a los siguientes criterios de inclusión: ser heterosexual, estar en una relación de convivencia de matrimonio, concubinato o unión libre de al menos un año de duración; los criterios de exclusión implican que el instrumento debe ser contestado en su totalidad y dar su consentimiento para el uso de la información.

\section{Material}

Se aplicó la escala de satisfacción marital de Roach et al. (1981), traducida al español y validada en población mexicana por Nina-Estrella (1985), con una confiabilidad alfa de 0.90. No se realizó ninguna adecuación a la escala; sólo se incluyeron los siguientes datos sociodemográficos: edad, género, escolaridad, número de años de casados o en convivencia, y número de hijos, para establecer posteriormente la comparación entre variables.

La escala consta de 48 ítems en forma de enunciados y mide la satisfacción marital a partir de la percepción que hace un individuo de su propio matrimonio a lo largo de un continuo, de mayor a menor favorabilidad en cierto tiempo (Roach et al., 1981). Para analizar los resultados de la escala, los enunciados se plantean dicotómicamente: satisfacción marital e insatisfacción marital. Los ítems determinados para indagar la satisfacción marital son 25 , correspondiendo éstos a los aspectos positivos y satisfactorios que se presentan en la relación ("yo sé lo que mi esposa(o) espera de nuestro matrimonio", "realmente me interesa mi esposo(a)", "estoy definitivamente satisfecho(a) con mi matrimonio"). En éstos se ofrecen cinco opciones de respuesta tipo Likert: totalmente de acuerdo equivale a una calificación de 5 puntos; de acuerdo, 4 puntos; indeciso, 3 puntos; en desacuerdo, 2 puntos; y totalmente en desacuerdo, 1 punto. Por otra parte, para la insatisfacción marital se evaluó con 23 ítems, los cuales refieren a aspectos negativos e insatisfactorios ("mi esposa(o) podría hacerme la vida más fácil si quisiera", "mi esposa(o) me exige injustamente que me ocupe de ella (o él) en mi tiempo libre", "mi esposa(o) no escucha lo que le digo"), ofreciendo de igual mane- ra cinco opciones de respuesta tipo Likert: totalmente de acuerdo corresponde a una calificación de 1 punto; de acuerdo, 2 puntos; indeciso, 3 puntos; en desacuerdo, 4 puntos; $y$ totalmente en desacuerdo, 5 puntos.

Para obtener la puntuación final se suman las respuestas de cada uno de los enunciados. Para efectos de codificación del resultado total del puntaje, y con la finalidad de una interpretación más sencilla, en esta investigación se siguió el método propuesto por Arias (1989), en donde el total de puntos (240 máximo y 48 mínimo) se divide entre 5, obteniendo puntajes entre 0.9 y 4.8, correspondiendo a la lógica de que a mayor puntuación mayor satisfacción marital.

\section{Procedimiento}

La escala fue aplicada en dos modalidades: en grupos (34 participantes) y de forma individual, en lugares libres y diversos, como consultorios, negocios y hogares (66 participantes). En ambos casos la instrucción se dio de manera verbal y escrita: "Marque con una X la casilla que refleje de forma aproximada lo que piensa acerca de la frase". Todos participaron de forma voluntaria y autorizaron que la información obtenida fuera empleada para su análisis, con la condición de que fuera totalmente confidencial y anónima. El tiempo aproximado en cada aplicación fue de 15 minutos.

\section{Análisis de datos}

Con la finalidad de realizar el análisis de datos se creó una base de los mismos en el Programa Estadístico SPSS versión 20.0 para Windows. Para obtener el grado de satisfacción marital se sumaron las respuestas por cada participante y se dividieron entre cinco; como se mencionó anteriormente, a mayor puntuación mayor satisfacción marital. Los grupos de cada factor sociodemográfico se conformaron de la siguiente forma: edad (17 a 23, 24 a 30,31 a 37,38 a 44, 45 a 51 y 52 a 59 años), género (hombre-mujer), escolaridad (educación básica, educación media superior y educación superior), número de años de casados ( 1 a 5, 6 a 10, 11 a 15, 16 a 20, 21 a 25, 26 a 30 y 31 a 33); número de hijos (0, 1, 2, 3 o más). Esta conformación se realizó con base en la regla de Sturges, es decir, se calculó el número de intervalos correspondiente a cada factor sociodemográfico, de modo que fueran conceptualmente adecuados, con el propósito de controlar posibles errores en la comparación de medias, el control de la normalidad de la muestra y el control de la varianza. Para el análisis de datos se llevó a cabo un ANOVA de un factor. Para los factores que revelaron una diferencia significativa se aplicó la prueba post hoc de Turkey al nivel de 0.05 \% de significación. 


\section{Resultados}

El promedio de edad en esta muestra fue de 36 años, el de tiempo de vivir en convivencia de 12.79 años, y el promedio de hijos fue de 2 . El $42 \%$ de los participantes cursó la educación básica, 23\% la educación media superior, 32\% la educación superior, y 3\% no proporcionó esta información.

En cuanto a los resultados arrojados por el ANOVA de un factor, se encuentra que, en cuanto a la variable de género, los hombres $(x A ́=4.68)$ reportan mayor satisfacción que las mujeres $(x \hat{A}=4.09)$, no siendo significativa la diferencia entre ambas medias ( $\mathrm{gl}=1, \mathrm{p}>0.05)$, es decir, estadísticamente tanto hombres como mujeres se encuentran igual de satisfechos con su relación. En cuanto a la variable de la edad, los sujetos que presentan mayor satisfacción marital son los que se encuentran entre los 24 y 30 años ( $x \hat{A}=$ 4.16), y los que presentan una mayor insatisfacción los que se ubican entre los 17 y 23 años ( $x A=3.69)$; al igual que en la variable anterior, al realizar la comparación de medias no se encuentra una diferencia estadísticamente significativa entre ambas ( $g l=5, p>0.05)$, por que se puede considerar que, indistintamente de la edad, todos los grupos analizados presentan estadísticamente una satisfacción marital similar. En relación con el número de años de casados, la media de satisfacción mayor se encuentra entre los sujetos de 31 a 33 años ( $x A ́=4.25$ ) y la mayor insatisfacción entre los de 26 a 30 años de casados ( $x \hat{A}=43.56)$, no obteniendo una diferencia de medias significativa $(g \mid=6, p>0.05)$, considerando con esto que los años no influyen en la satisfacción marital. Atendiendo al número de hijos, los participantes mostraron una puntuación media mayor cuando se tiene sólo un hijo (xÁ =4.33), a diferencia de quienes tienen de tres a más hijos ( $\mathrm{xÁ}$ =3.78), no encontrando diferencias estadísticamente significativas entre ambos grupos ( $\mathrm{gl}=3, \mathrm{p}>0.05)$. En este último caso, y al igual que en las variables anteriores, el número de hijos no influye en la satisfacción marital.

Por último, al hacer el análisis de medias se encuentra que los sujetos con escolaridad de educación básica ( $x A ́=3.81)$ son los que presentan mayor insatisfacción en su relación, seguidos de los que cursaron educación media superior ( $x A ́=3.83)$, siendo los más satisfechos en su relación los sujetos que han cursado la educación superior ( $x A ́$ =4.38). En este caso, al efectuar la comparación de medias se encuentra que no existen diferencias estadísticamente significativas entre el grupo de educación básica y educación media superior ( $p>0.05$ ); sin embargo, al realizar la comparación de medias de educación básica, educación media superior y educación superior, se encuentra que la educación superior es estadísticamente más satisfecha en relación con las anteriores $(\mathrm{gl}=2, \mathrm{p}<$ 0.05); en este caso se puede sugerir que el mayor nivel de satisfacción marital lo reportan los participantes con un nivel educativo superior (tabla 1 y figura 1).

\section{Tabla 1.}

Análisis comparativo de factores sociodemográficos (sexo, edad, escolaridad, número de años casados y número de hijos) vs. satisfacción marital

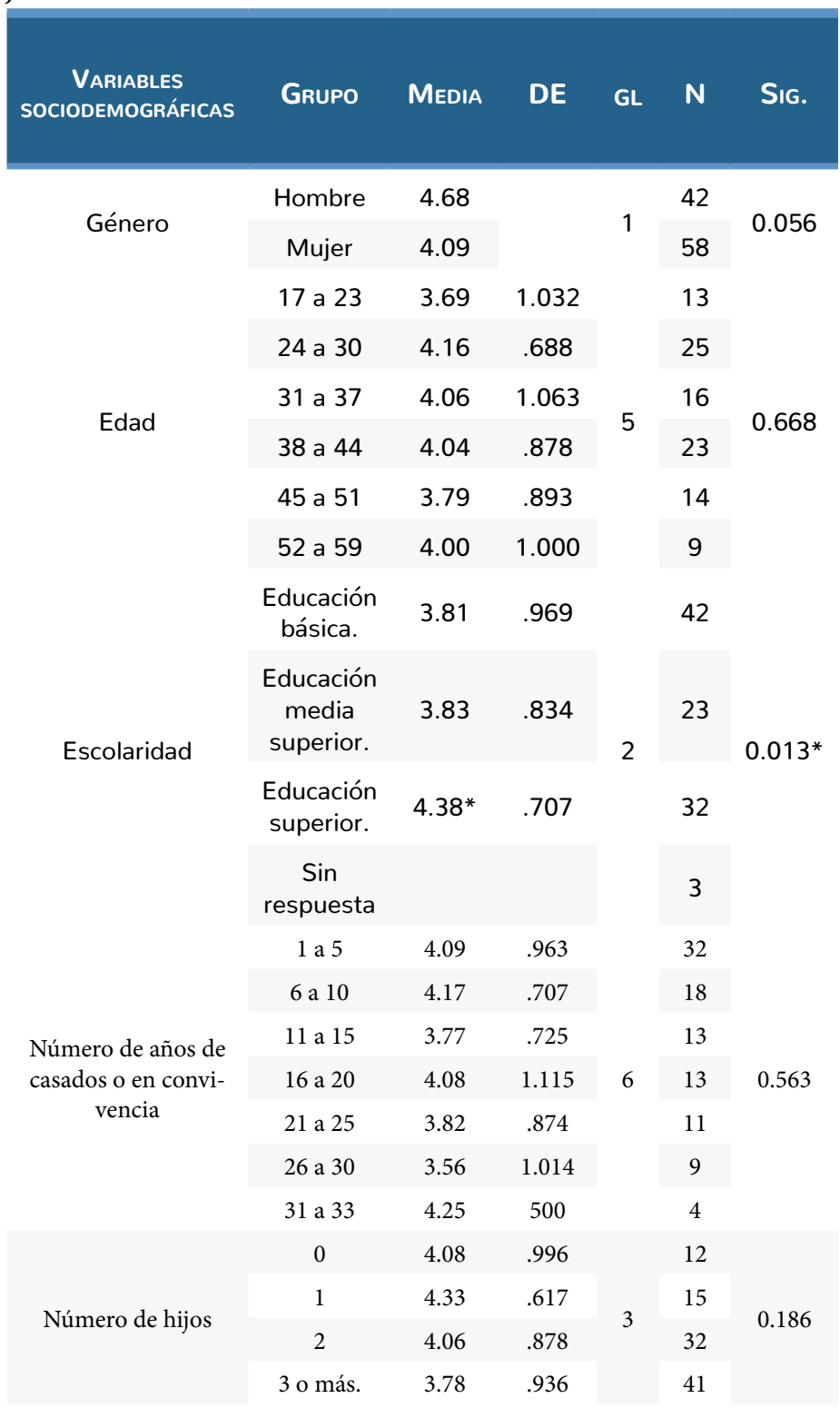

* La variable de escolaridad es la única que muestra una diferencia estadísticamente significativa ( $p>0.05$ ); es decir, a mayor grado académico mayor satisfacción marital.

\section{Discusión}

De acuerdo con los resultados obtenidos no se presentaron diferencias estadísticamente significativas entre los factores sociodemográficos de género, edad, número de años de casados y número de hijos en relación con la 
satisfacción marital; el único factor donde encuentran diferencias significativas es en la escolaridad. En este sentido puede observarse que tanto hombres como mujeres presentan una satisfacción similar en su relación, coincidiendo con los hallazgos reportados por Ottu y Akpan (2011) y Bounchard (2013), quienes señalan que aun cuando el rol de género contribuye a los conflictos maritales, las comparaciones de medias no son significativas. Es importante señalar que aunque no es significativo, los datos presentan una ligera tendencia a que los hombres están más satisfechos que las mujeres, lo sugiere que, como señalaron Pick y Andrade (1988) y Lavner y Bradbury (2010), las mujeres suelen presentar cierta insatisfacción generada por los roles de género asignados por el contexto en que viven.

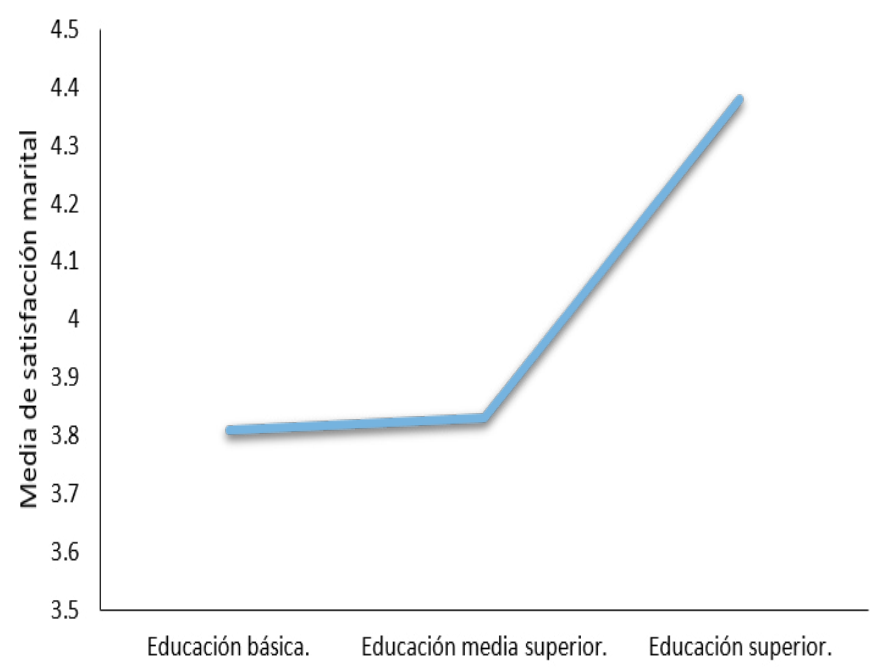

Figura 1.

Puntajes medios obtenidos en el factor socioeconómico escolaridad en relación con la satisfacción marital.

En cuanto al factor sociodemográfico de la edad, y aunque no son significativas las diferencias, el índice más bajo se ubica en el grupo de edad de 17 a 23 años, lo cual coincide con lo señalado por Campuzano (2002) en relación con que son las parejas más jóvenes quienes suelen presentan mayor insatisfacción marital, esto debido probablemente a conflictos debidos a la inmadurez y a las altas expectativas que se tienen puestas en el otro. En este mismo sentido, y atendiendo lo propuesto por Vera et al. (2001) y Martínez (2004), se puede considerar que a mayor edad se presentará mayor satisfacción marital, porque hay mayor conciencia de los problemas familiares y maritales, y por tanto se ocupan en buscar soluciones para éstos. En el caso del grupo de entre 24 y 30 años, son quienes presentan mayor satisfacción marital en relación con los demás; con esto rompe la idea de una lineal ascendente y progresiva de satisfacción, condición sugerida por estos mismos autores.

Sumado a lo anterior, los datos no muestran el patrón de satisfacción propuesto por Kimble (2002), quien postula que la satisfacción marital puede presentarse en forma de " $U$ " al transcurrir los años de casados o de estar juntos (satisfacción-insatisfacción-satisfacción); por su parte, Pick y Andrade (1988) plantean que la satisfacción tiene un descenso progresivo lineal donde pocas veces se vuelve a elevar este concepto; o según el modelo de Rivera y Heresi (2011), en el cual se traza un patrón lineal ascendente, a razón de que conforme aumentan los años de matrimonio aumenta la satisfacción marital. Los datos de esta investigación, adquieren su propio patrón, considerando con esto que el contexto de la muestra estudiada, en este caso semiurbano, influye en la satisfacción marital y su percepción al transcurrir de los años de convivencia.

Los resultados obtenidos entre la cantidad de hijos y su relación con la satisfacción marital, coincide con lo dicho por Shapiro et al. (2000), los cuales sugieren que a mayor cantidad de hijos se genera mayor malestar en uno o en ambos miembros de la pareja. Como se muestra en los resultados de este estudio, las parejas con tres o más hijos son las más insatisfechas, y las parejas con un hijo las más satisfechas. Parece que, al igual que en los contextos urbanos — e incluso extranjeros-, los matrimonios considerados en este ámbito semiurbano buscan mejorar la organización, dedicación y tiempo para ellos, así como disminuir esfuerzos, dedicación y economía (Pick y Andrade, 1988; Mora et al., 2013; Sandler, 2013).

Por último, la relación escolaridad y satisfacción marital no muestra diferencias significativas entre los sujetos con educación básica y educación media superior; para esta muestra, el nivel de satisfacción marital es similar. Sin embargo, hay una diferencia significativa entre las medias de educación básica y educación media superior en relación con la escolaridad de nivel superior; esto es, a mayor escolaridad mayor satisfacción marital, lo que puede explicarse considerando los hallazgos de investigaciones anteriores, donde se encontró que las personas con bajo nivel escolar suelen desempeñar trabajos de poco prestigio que les redituan ingresos insuficientes para solventar las necesidades básicas de la familia, lo que eventualmente causa insatisfacción marital; y en caso contrario, las personas que perciben un ingreso más alto son las que tienen una educación profesional, cuyo efecto es una satisfacción marital alta (Campbell et al., 1976; Vera, 2011). Por otro lado, Arias (1989) explica que existe mayor probabilidad de que los sujetos con nivel educativo medio realicen una comparación con los sujetos con mayor pre- 
paración profesional, generando una insatisfacción marital como reflejo de una insatisfacción general.

\section{Conclusiones}

La satisfacción marital es un fenómeno multicausal y podría estar determinada por el contexto sociocultural; algunos de los resultados de este estudio no coinciden con lo expuesto por investigaciones previas (Kimble, 2002; Pick y Andrade, 1988; Rivera y Heresi, 2011), debido a que éstas se han llevado a cabo en contextos urbanos, y en este caso la investigación se realizó en un ámbito semiurbano. Asimismo, la satisfacción marital puede aumentar o disminuir según las circunstancias por que esté pasando la pareja, la edad de cada individuo, tener hijos o no, el número de hijos en caso de tenerlos, el grado escolar, el rol de género que se asigna culturalmente, e incluso los años de casados, determinan cómo un individuo valora su relación marital en un momento dado y según el contexto en que vive. Se detectó que el instrumento de medición empleado remite a una valoración de la satisfacción marital de forma general, sin considerar aspectos más específicos de la vida de una pareja; por ello, se considera necesario adaptar o crear nuevos instrumentos que permitan indagar los pormenores del universo de las relaciones de pareja.

\section{Referencias}

Arias, F. (1989) Una investigación sobre la escala de satisfacción marital. Revista Latinoamericana de Psicología. 21(3): 423-436.

Armenta C., Sánchez, R. y Díaz, R. (2012). ¿De qué manera el contexto afecta la satisfacción con la pareja? Suma Psicológica. 19 (2): 51-62.

Armenta, C. y Díaz-Loving, R. (2008). Comunicación y Satisfacción: Analizando la interacción de pareja. Psicología Iberoamericana. 16(1): 23-27.

Bradley, N. (1999). Sampling for internet surveys. An examination of respondent selection for internet research. Journal of the Market Research Society. 41 (4): 387-395.

Becerra, S., Roldan, W. \& Flores, W. (2012). Factores de enriquecimiento, mantenimiento, comunicación y bienestar en la relación marital. Revista de Psicología GEPU. 3(1): 37-62.

Bounchard, G. (2013). How do parents react when their children leave home? An integrative review. Journal of Adult Development. 21(2): 69-79.

Campbell, A., Converse, P., \& Rodgers, W. (1976). The Quality of American life: Perceptions, Evaluations and Satisfactions. Estados Unidos: Russell Sage Foundation. 591 Pp.

Campuzano, M. (2002). La pareja humana: Su psicología, sus conflictos, su tratamiento. México: Plaza y Valdés. 171 Pp.
Castro, J. P. (2014). Es preocupante alza de divorcio: Pérez, en Página 24. [En línea]. Disponible en: http:// pagina24zacatecas.com.mx/local/2014/04/24/espreocupante-alza-de-divorcios-perez. Fecha de consulta: 12 de enero de 2015.

Covarrubias, M. \& Gómez, J. (2012). Transformación y contemporaneidad de la familia: el caso de las familias mexicanas. Revista de Psicología Perú. 14(2): 244-259.

Dainton, M., Stafford, L., \& Canary, D. (1994). Maintenance Strategies and Physical Affection as Predictors of Love, Liking, and Satisfaction in Marriage. Communication Reports. 7(2): 88-97.

Instituto Nacional de Estadística y Geografía (2014). Estadísticas a propósito del Día internacional de la eliminación de la violencia contra la mujer. Datos nacionales. [En línea]. Disponible en: http://www.inegi.org.mx/est/contenidos/ proyectos/ce/ce2014/default.aspx?bi=1. Fecha de consulta: 15 de diciembre de 2014.

Instituto Nacional de Estadística y Geografía (2015). Estadística de nupcialidad. Datos nacionales. [En línea]. Disponible en: http://www.inegi.org.mx/inegi/default. aspx?s=est $\& c=11093$. Fecha de consulta: 9 de septiembre de 2015.

Kimble, Ch. (2002). Psicología social de las Américas. México: Pearson Educación. 326 Pp.

Lavner, J. \& Bradbury, T. (2010). Patterns of Change in Marital Satisfaction over the Newlywed Years. Journal of Marriage and Family. 72(5): 1171-1187.

López, P. \& García, E. (2005). Aristóteles política. [En línea]. Disponibleen: https://books.google.com.mx/books?id=c5l hK5RKy8MC\&printsec=frontcover\&dq=La+pol\%C3\%ADtic $a+$ Arist\%C3\%B3teles\&hl=es-419\&sa =X\&ei=d2W1VL6-I4uv yASt64LIBw\&ved=0CCAQuwUwAA\#v=onepage\&q=La\%20 pol\%C3\%ADtica\%20Arist\%C3\%B3teles\&f=false. Fecha de consulta: 20 de diciembre de 2014.

Martínez, S. (2004). Efectos de la edad, sexo y la escolaridad en la satisfacción marital, en Revista Episteme. [En línea]. Disponible en: http://www.uvmnet.edu/investigacion/ episteme/numero1-04/reportes/a_efectos.asp\#autor. Fecha de consulta: 15 de diciembre de 2014.

Mora, M., Gómez, M. \& Rivera, M. (2013). La satisfacción marital y los recursos psicológicos en las parejas con y sin hijos pequeños en pro del bienestar familiar. Revista de Psicología. 10(22): 79-96.

Nina-Estrella, R. (1985). Autodivulgación y satisfacción marital en matrimonios de México y Puerto Rico (tesis de Maestría). Universidad Nacional Autónoma de México, México, D.F.

Ojeda, N. \& González, E. (2008). Divorcio y separación conyugal en México en los albores del siglo XXI. Revista Mexicana de Sociología. 70 (1): 111-145.

Ottu, I. \& Akpan, U. (2011). Predicting Marital Satisfaction from the Attachment Styles and Gender of a Culturally and Religiously Homogenous Population. Gender y Behavior. 9(1): 36-56.

Pick, A. \& Andrade, P. (1988). Diferencias sociodemográficas en la satisfacción marital: el caso de México. Revista de Psicología Social. 3(1): 91-98.

Rivera, D. \& Heresi, E. (2011). Integración de la teoría del apego 
y modelos basados en la evidencia en la comprensión de la satisfacción marital, en Revista argentina de clínica psicológica. [En línea]. Disponible en: http://dialnet. unirioja.es/servlet/articulo?codigo $=4260404$. Fecha de consulta: 8 de diciembre de 2014.

Roach, J., Frazier, P., \& Bowden, R. (1981). The Marital Satisfaction Scale: Development of Measure for Intervention Research. Journal of Marriage and the Family. 43(3): 537-546.

Sandler, L. (2013). Having it all Without Having Children, in Magazine Time. [En línea]. Disponible en: http://time. com/241/having-it-all-without-having-children/. Fecha de consulta: 1 de diciembre de 2014.

Shapiro, A., Gottman, J., \& Carrere, S. (2000). The Baby and the Marriage: Identifying Factors that Buffer Against Decline in Marital Satisfaction after the First Baby Arrives. Journal of Family Psychology. 14(1): 59-70.

Snyder, D. (2008). Inventario de satisfacción marital-Revisado (MSI-R). México: Manual Moderno.
Stewart, M. (2012). The Impact of Length of Relationship on Conflict, Communication and Relationship Satisfaction in Couples. Graduate Theses and Dissertations. 12474: 1-36.

Vera, J. (2011). Patrones de comunicación en parejas y su relación con la satisfacción marital en profesores de la Universidad privada de Asunción. Eureka. 8(2): 200-216.

Vera, J., Félix-Castro, J. y Rodríguez-Barreras, A. (2001). Satisfacción marital, edad, número de hijos y años de pareja, en Revista de la Facultad de Psicología de la Universidad Nacional Mayor de San Marcos. [En línea]. Disponible en: http://www.ciad.mx/archivos/desarrollo/ publicaciones/PUBLICACIONES/Produccion $\% 20$ Academica/Articulos/2001/82.pdf. Fecha de consulta: 15 de diciembre de 2014.

Wilson, J. (1998). Sorpresa en los investigadores maritales: finalmente, ¿qué patrones comunicacionales son disfuncionales? Revista de Psicología de la Universidad de Chile, 8, 105-115. 


\section{Meta-Análisis del Artículo}

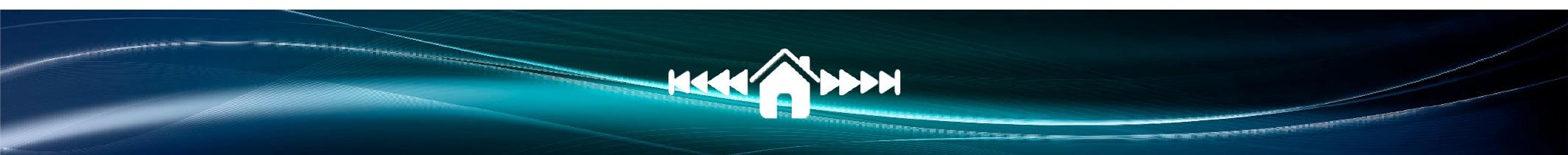


Revista Digital Internacional de Psicología y Ciencia Social |V Vol. 2 | Núm. 2 | Jufio-Diciembre 2016 | ISSN 1405-5802

\section{Dimensión Cuantitativa}

\section{Perfil de Evaluación entre pares}
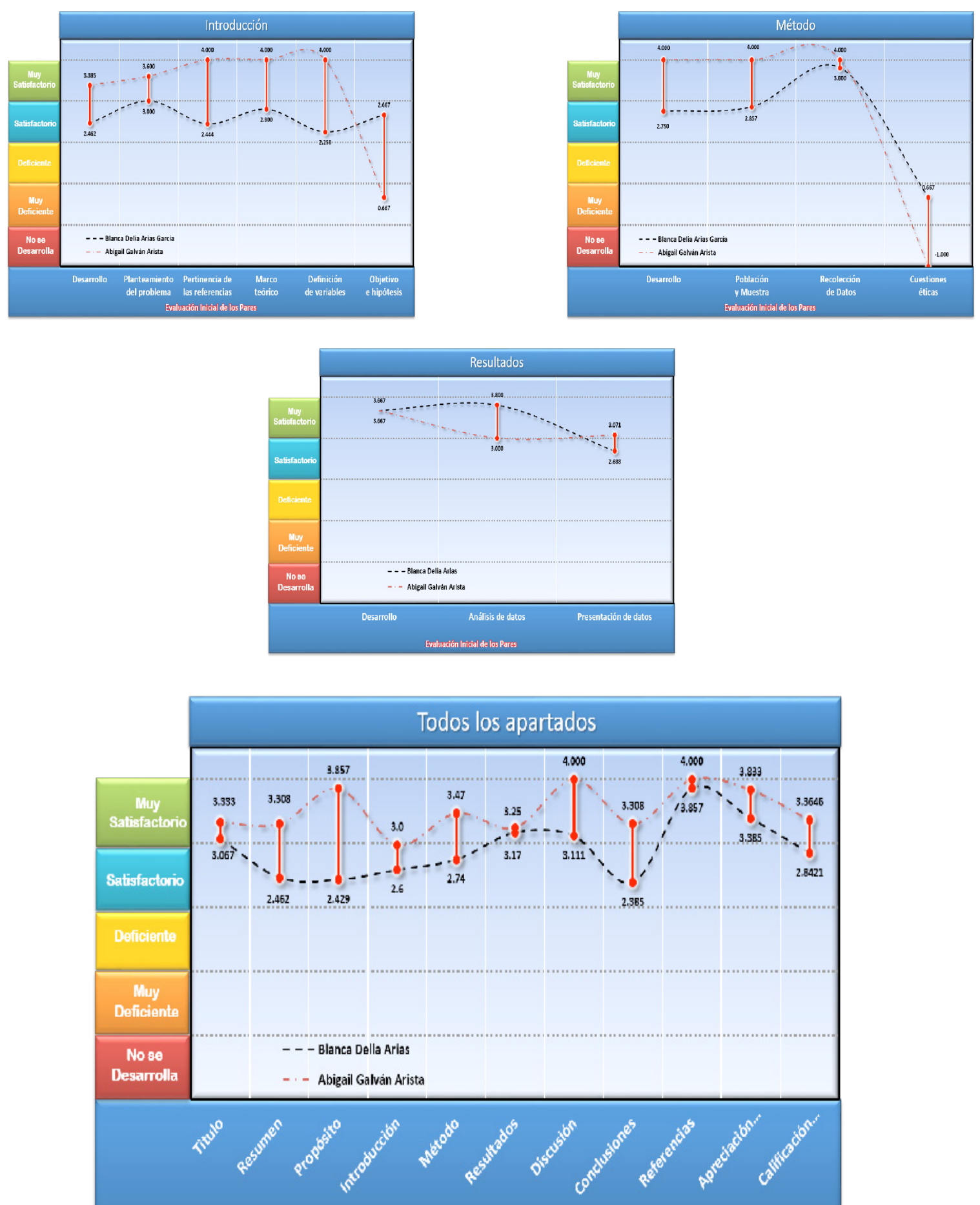

Evaluación Inicial de los Pares 


\section{Índice de Concordancia}

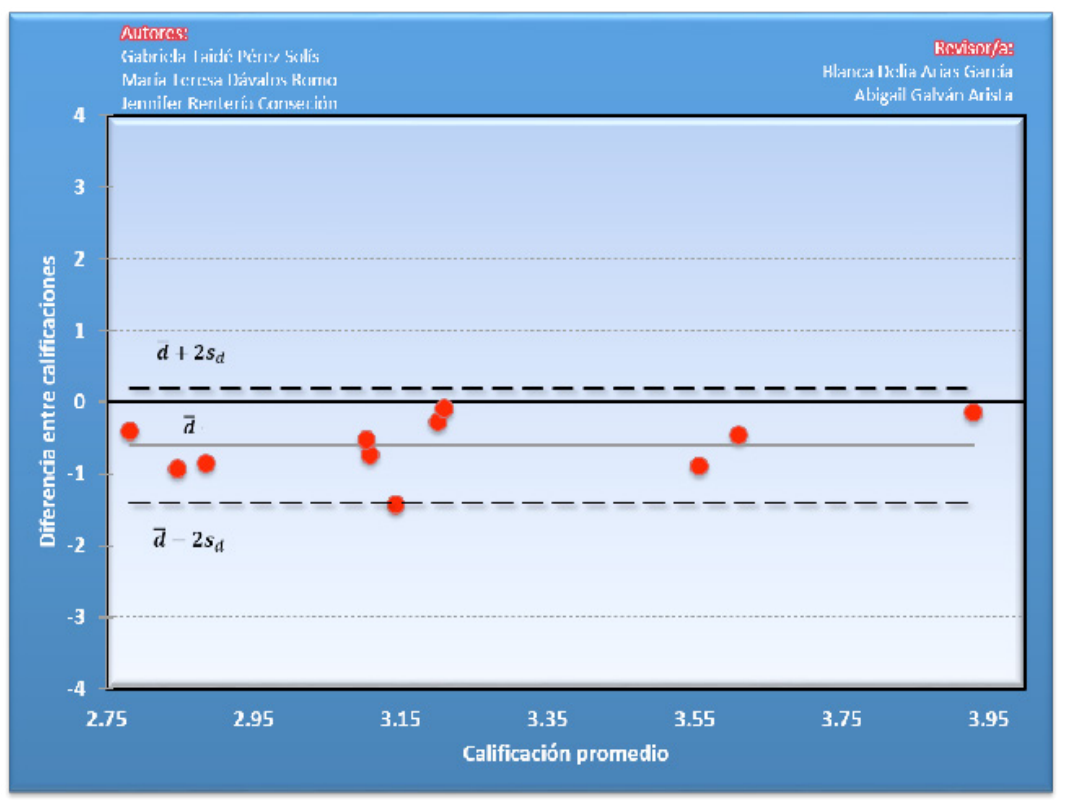

Índice de Acuerdo
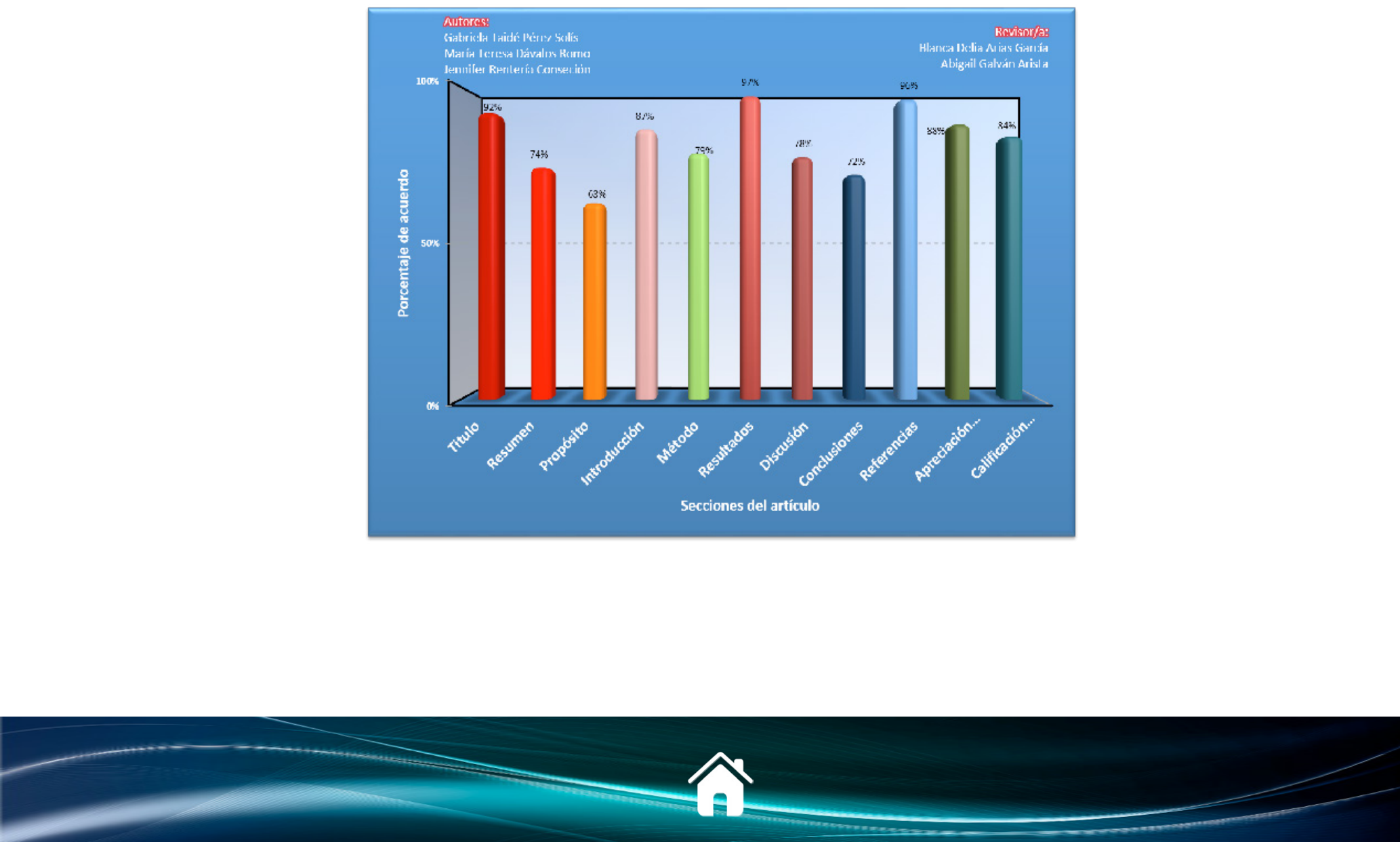
Dimensión Cualitativa

\begin{tabular}{|l|l|}
\hline \multicolumn{1}{|c|}{ Revisor 1 } & Revisor 2 \\
\hline \multicolumn{1}{|c|}{ Blanca Delia Arias García } & Abigail Galvan Arista \\
\hline \multicolumn{1}{|c|}{ Título/Autoría } \\
\hline $\begin{array}{l}\text { Si la única variable donde se encuentran diferencias es- } \\
\text { tadisticamente significativas es "nivel escolar" o "nivel } \\
\text { educativo" es recomendable que esté en el título, dado } \\
\text { que se sugiere que el título esté estrechalmente ligado al } \\
\text { hallazgo más importante del estudio. }\end{array}$ & $\begin{array}{l}\text { No se incluyen las instituciones de trabajo ni contacto } \\
\text { de las autoras }\end{array}$ \\
\hline $\begin{array}{l}\text { Esta afirmación parece muy determinista, como si la } \\
\text { insatisfacción marital fuera la única causa de los divor- } \\
\text { cios. Sugiero cuidar el alcance de esta tesis, por ejemplo } \\
\text { "La insatisfacción marital es un factor asociado del alto } \\
\text { índice de divorcios" Si se está trabajando con la prueba } \\
\text { de hipótesis podría ser más adecuado señalar "diferen- } \\
\text { cias estadísticamente significativas" Se sugiere utilizar } \\
\text { palabras diferentes en el título y en las palabras clave, } \\
\text { ya que la forma en la cual operan las bases de datos } \\
\text { para recuperar información, considera a titulo y a las } \\
\text { palabras clave y se duplicaría. }\end{array}$ & Excede las 150 palabras y no contiene datos numéricos \\
\hline $\begin{array}{l}\text { No queda claro si el propósito es asociar los factores so- } \\
\text { ciodemográficos o si hay diferencias estadísticamente } \\
\text { significas entre grupos. Es decir, buscarán correlación } \\
\text { o buscarán determinar a través de pruebas de hipótesis } \\
\text { si existen diferencias estadisticamente significativas al } \\
\text { comparar grupos, lo cual en las conclusiones se peude } \\
\text { derivar la conclusión de que hay grupos con algunas } \\
\text { características que prsentan mayor o menor satisfac- } \\
\text { ción marital en comparación con otros. }\end{array}$ & Bien redactado \\
\hline \begin{tabular}{l} 
Próposito del Estudio \\
\hline
\end{tabular} & \\
\hline
\end{tabular}




\begin{tabular}{|c|c|}
\hline Revisor 1 & Revisor 2 \\
\hline \multicolumn{2}{|c|}{ Introducción } \\
\hline $\begin{array}{l}\text { Se incluye un concepto legal como "separación de he- } \\
\text { cho", se recomienda especificar de forma concreta en } \\
\text { que consiste dado que está revista tiene mayor proba- } \\
\text { bilidad de ser leida por psicologos, sociologos, etc.Por } \\
\text { ejemplo,...por las separaciones de hecho, lo cual signifi- } \\
\text { ca que la pareja se encuentra formalmente viviendo de } \\
\text { forma separada sin que esto implique disolver el } \\
\text { matrimonio.... Me parece que falta una argumenta- } \\
\text { ción para especificar o justificar la relevancia del tema, } \\
\text { es decir, porque es importante hablar del divorcio y la } \\
\text { satisfacción marital? Brindan estadísticas, quedo claro } \\
\text { que es un problema con alta indicencia y muy común, } \\
\text { pero y por que es importante estudiarlo más allá de las } \\
\text { elevadas estadisticas? Esto tiene un impacto en las } \\
\text { familias? Cual? Tiene impacto en la educación de los } \\
\text { hijos? En los recursos que las familias obtienen de te- } \\
\text { ner ambos padres que aporten ingresos? Se asocia a de- } \\
\text { presión? A disfunción famliar?...etc etc. me parece que } \\
\text { falta argumentar un poco más allá de las estadísticas.N } \\
\text { No queda muy claro como es que la variable sociode- } \\
\text { mográfica tiene un impacto en la interacción en la } \\
\text { satisfacción, que señalan los autores al respecto? Con- } \\
\text { sidero que no termina de quedar claro la justificación } \\
\text { de la elección de variables: }{ }^{\star} \text { Si hay variables de orden } \\
\text { psicológico como la intimidad, compromiso, comuni- } \\
\text { cación, etc etc porque no abordar el fenómeno desde } \\
\text { estas variables en relación a la satisfacción marital, por- } \\
\text { que elegir variables demográficas? A la mejor valdria la } \\
\text { pena especificar la dimensión psicológica en relación } \\
\text { a las variables demográficas, es decir, ir más allá de va- } \\
\text { riables estructurales o demográficas para considerar } \\
\text { variables de proceso o más de orden psicológicas. Me } \\
\text { parece que el estudio de variables demográficas puede } \\
\text { aportar información relevante pero hay que engrosar la } \\
\text { justificación. Las variables sociodemográficas permiten } \\
\text { generar perfiles, conformar grupos de comparación o } \\
\text { análisis y de este punto se parte a la mejor para realizar } \\
\text { estudios de variables psicológicas o de procesos, pero } \\
\text { no queda muy clara la justificación de por qué estas va- } \\
\text { riables. }\end{array}$ & Falta profundizar en el modelo teórico y la hipótesis. \\
\hline
\end{tabular}




\begin{tabular}{|l|ll|}
\hline Revisor 1 & Revisor 2 \\
\hline & Método & \\
\hline
\end{tabular}

Consideren describir con claridad la clasificación diseño si es pre experimental, exploratorio, transversal, etc No dudo que se hayan considerado las cuestiones éticas, así que valdría la pena que las describan.

Falta el consentimiento informado

\section{Resultados}

Por favor consideren los comentarios específicos que encontrarán en el documento.

Los resultados no son claros y la lectura se vuelve complicada

\section{Discusión}

Se sugiere tener cuidado en el lenguaje que se usa pues el trabajo estima diferencias, no hace correlaciones, revisar en el documento los comentarios específicos.

Bastante claro

\section{Conclusiones}

Se sugiere señalar con claridad las contribuciones del estudio respecto a investigaciones antecedentes y citarlas.

Muy cortas las conclusiones, hay muchas cosas más en las que se puede profundizar como por ejemplo, mencionar como podrían resolverse a futuro las deficiencias de su estudio

\section{Referencias}

Se sugiere usar el \& en lugar de and. 
